\title{
Deep Transfer Learning for Source Code Modeling
}

\author{
Yasir Hussain ${ }^{\mathrm{a}, *}$, Zhiqiu Huang ${ }^{\mathrm{a}, \mathrm{b}, \mathrm{c}, *}$, Yu Zhou ${ }^{\mathrm{a}}$, Senzhang Wang ${ }^{\mathrm{a}}$ \\ ${ }^{a}$ College of Computer Science and Technology, Nanjing University of Aeronautics and \\ Astronautics (NUAA), Nanjing 211106, China \\ ${ }^{b}$ Key Laboratory of Safety-Critical Software, NUAA, Ministry of Industry and Information \\ Technology, Nanjing 211106, China \\ ${ }^{c}$ Collaborative Innovation Center of Novel Software Technology and Industrialization, \\ Nanjing 210093, China
}

\begin{abstract}
In recent years, deep learning models have shown great potential in source code modeling and analysis. Generally, deep learning-based approaches are problemspecific and data-hungry. A challenging issue of these approaches is that they require training from starch for a different related problem. In this work, we propose a transfer learning-based approach that significantly improves the performance of deep learning-based source code models. In contrast to traditional learning paradigms, transfer learning can transfer the knowledge learned in solving one problem into another related problem. First, we present two recurrent neural network-based models RNN and GRU for the purpose of transfer learning in the domain of source code modeling. Next, via transfer learning, these pre-trained (RNN and GRU) models are used as feature extractors. Then, these extracted features are combined into attention learner for different downstream tasks. The attention learner leverages from the learned knowledge of pre-trained models and fine-tunes them for a specific downstream task. We evaluate the performance of the proposed approach with extensive experiments with the source code suggestion task. The results indicate that the proposed approach outperforms the state-of-the-art models in terms of accuracy, precision, recall, and F-measure without training the models from scratch.
\end{abstract}

Keywords: Transfer Learning, Deep Neural Language Models, Source Code Modeling, Attention Learning.

\section{Introduction}

Source code suggestion and syntax error fixing are vital features of a modern integrated development environment (IDE). These features help software developers to build and debug software rapidly. Recently, deep learning-based

\footnotetext{
${ }^{*}$ Corresponding author

Email addresses: yaxirhuxxain@nuaa.edu.cn (Yasir Hussain), zqhuang@nuaa.edu.cn (Zhiqiu Huang), zhouyu@nuaa.edu.cn (Yu Zhou), szwang@nuaa.edu.cn (Senzhang Wang)
}

Preprint submitted to Arxiv 
language models have shown great potential in various source code modeling tasks [3, 5, 46, 20, 28, 16, 43, 47, 56, 27. Several studies have explored deep learning for source code suggestion [43, 56] in which they suggest the next possible source code token. They take a fixed size context prior to the prediction position as features and help the software developers by suggesting the next possible code token. Further, deep learning has recently been explored for syntax error detection and correction [46, 20] problem. They consider the source code syntax as features and use them for the correction of the syntax errors found in a source code file. Moreover, deep learning has shown its effectiveness in the source code summarization [3, 28, 16], which summarizes the working of source code.

A challenging issue of these approaches is that they are problem-specific which requires training from starch for a different related problem. Further, deep learning-based approaches are data-hungry which means they require training on large data set to produce satisfactory results. Furthermore, deep learning models requires days to train while training on a large dataset. To overcome these issues, we exploit the concept of transfer learning in this work. In transfer learning, the learned knowledge from a pre-trained model is extracted and then be used for a similar downstream task [45].

This work proposes a transfer learning-based approach that significantly improves the performance of deep learning-based source code models. First, we exploit the concept of transfer learning for deep learning-based source code language models. The key idea is to use a pre-trained source code language model to transfer the learned knowledge from it to a different related problem. We train two different variants of recurrent neural network-based models RNN and GRU for the purpose of transfer learning. Then, we combine the learned knowledge of pre-trained (RNN and GRU) models into attention learner for a downstream task. The attention learner leverage from the learned knowledge of pre-trained models and fine-tunes it for a specific downstream task. Via transfer learning, pre-trained models are used to extract generalized features and then fine-tune them for a target task without requiring the model training from scratch. We evaluate the proposed approach with the downstream task of source code suggestion.

This work makes the following unique contributions:

- We exploit the concept of transfer learning in the domain of source code. We propose transfer learning-based attention learner approach for the downstream task of source code suggestion.

- We present two recurrent neural network-based (RNN and GRU) pretrained models for the purpose of transfer learning in the domain of source code.

- An extensive evaluation of the proposed approach on the real-world data set shows significant improvement in the state-of-the-art language models. 


\section{Related Work}

In this section, we present background study on deep learning, transfer learning and source code language models.

\subsection{Source Code Modeling}

Hindle et al. 23. have shown how natural language processing techniques can help in source code modeling. They provide a $n$-gram based model which helps predict the next code token in Eclipse IDE. Tu et al. [51, proposed a cachebased language model that consists of an n-gram and a cache. Hellendoorn et al. 22, further improved the cache-based model by introducing nested locality. Another approach for source code modeling is to use probabilistic context-free grammars(PCFGs) [7. Allamanis et al. 1] used a PCFG based model to mine idioms from source code. Maddison et al. [35] used a structured generative model for source code. They evaluated their approach with n-gram and PCFG based language models and showed how they can help in source code generation tasks. Raychev et al. 44 applied decision trees for predicting API elements. Chan et al. 8] used a graph-based search approach to search and recommend API usages.

Recently there has been an increase in API usage [54, 30, 14] mining and suggestion. Thung et al. 50] introduced a recommendation system for API methods recommendation by using feature requests. Pham et al. [40] proposed a methodology to learn API usages from byte code. Hussain et al. [26] proposed GRU based model for source code suggestion and completion task (completion of a whole line of code). A neural probabilistic language model introduced in [2] that can suggest names for the methods and classes. Franks et al. [17] created a tool for Eclipse named CACHECA for source code suggestion using a $n$-gram model. Nguyen et al. 37] introduced an Eclipse plugin which provide code completions by mining the API usage patterns. Chen et al. 9] created a web-based tool to find analogical libraries for different languages.

A similar work conducted by Rabinovich et al. [41, which introduced an abstract syntax networks modeling framework for tasks like code generation and semantic parsing. Sethi et al. [47] introduced a model which automatically generate source code from deep Learning-based research papers. [4, Allamanis et al. proposed a bimodal to help suggest source code snippets with a natural language query. Recently deep learning-based approaches have widely been applied for source code modeling. Such as code summarization [28, 3, 19, 29, code mining [57], clone detection [33, 61], API learning [18, 55], code generation 62 etc.

We observe [43, 56] approaches are related to our downstream task of source code suggestion. Raychev et al. [43, used RNN for the purpose of code completion specifically focusing on suggesting source code method calls. Similarly, White et al. [56] applied RNN based deep neural network for source code completion task. Generally, these approaches [43, 56] are problem-specific which requires training from scratch for a different related problem. In this work, we exploit the concept of transfer learning to extract the learned knowledge from 
pre-trained models and then fine-tunes it for a related problem, which shows a significant performance boost, without requiring the models training from scratch.

\subsection{Transfer Learning}

Transfer learning as the name suggests intending to transfer knowledge (features) learned in solving one problem into another related problem. Hu et al. 24. have proposed a transfer metric learning approach for visual recognition in cross-domain datasets. Duan et al. [15] have proposed a kernel learning approach for the detection of cross-domain keyframe feature changes. Pan et al. [39] have proposed a dimensionality reduction method which uses the transfer learning approach by minimizing the distance between distributions between target and source domains. Khan et al. 31] have proposed a deep transfer learning approach for the detection of breast cancer by using pre-trained GoogLeNet, VGGNet, and ResNet. Huang et al. [25] have proposed a transfer learning-based approach for Synthetic Aperture Radar (SAR) classification with limited labeled data. Kraus et al. 32 proposed a decision support system by using deep neural networks and transfer learning for financial disclosures. Further, transfer learning has been extensively studied for various tasks in the field of image and text classification [48, 60, 59, 32. In this work, we exploit the transfer learning approach for the purpose of source code modeling. Instead of using a single model for transferring knowledge, in this work, we use a novel approach that transfers knowledge from two different recurrent neural networkbased pre-trained models and then fine-tunes it by using attention learner for a specific source code modeling tasks.

\section{Preliminary}

In this section, we will discuss the preliminaries and technical overview of this work.

\subsection{Recurrent Neural Network}

The recurrent neural network has recently shown great potential in a wide range of applications including image recognition, text classification, and source code modeling. However, a major drawback of vanilla RNN is the vanishing gradient which can be overcome by using the gated recurrent unit (GRU) [58]. The GRU exposes the full hidden content on each timestep, thus evading the disappearing gradient issue. It can be expressed as

$$
\begin{gathered}
h_{i}=\left(1-z_{i}\right) h_{i-1}+z_{i} \hbar_{i} \\
z_{i}=\phi\left(W_{z} \tau_{i}+U_{z} h_{i-1}\right) \\
\hbar_{i}=\tanh \left(W \tau_{i}+r_{i} \otimes U h_{i-1}\right) \\
r_{i}=\phi\left(W_{r} \tau_{i}+U_{r} h_{i-1}\right)
\end{gathered}
$$




\subsection{Attention Learner}

Recently, attention-based approaches have shown great potential in various fields such as speech recognition [11, machine translation [34, 6], and more [52, 5]. The attention ([6]) model calculates a context vector as the weighted mean of the state sequence. it can be expressed as

$$
\begin{gathered}
a_{i}^{t}=\frac{\exp \left(c_{i}^{t}\right)}{\sum_{j=1}^{T} \exp \left(c_{j}^{t}\right)} \\
c_{i}^{t}=a\left(s^{t-1}, h_{i}\right)
\end{gathered}
$$

\section{Proposed approach}

This section discusses the proposed approach in detail. The Fig. 1 shows the overall workflow of the proposed approach. This section is subdivided into two major parts. In the first part, we discuss the preparation of pre-trained models for the purpose of transfer learning. The second part discusses the key steps involved to prepare the source code for the downstream task of source code suggestion. Further, we discuss the attention learner, which leverages the learned knowledge from pre-trained models and fine-tunes it for the source code suggestion task. The details about each step are given in the following subsections.

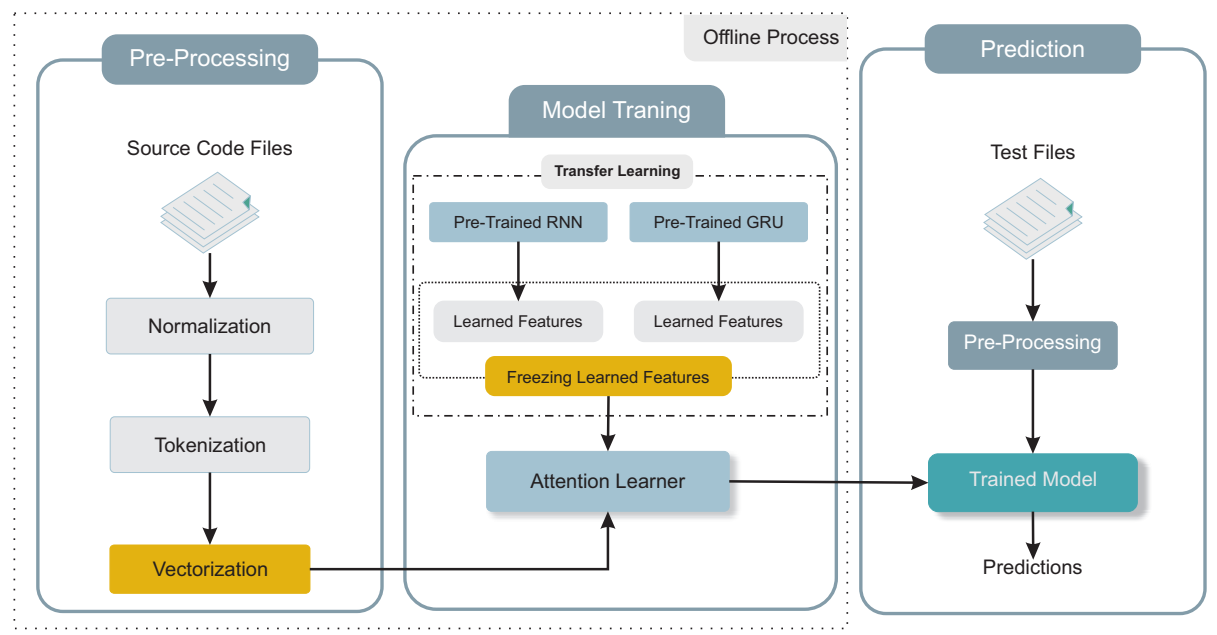

Figure 1: Overall framework of the proposed approach

\subsection{Transfer Learning}

Fig. 2 shows the difference between traditional learning and transfer learningbased approaches for source code modeling. For the purpose of transfer learning, we first need a pre-trained model. There are several CNN [49, 21] and 


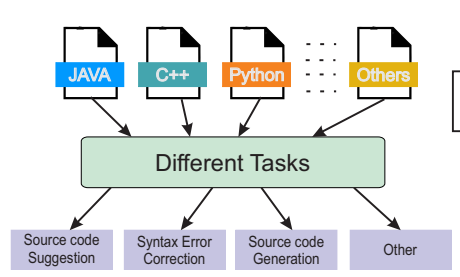

(a)

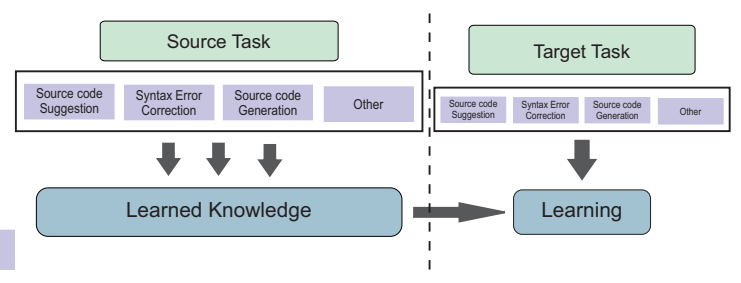

(b)

Figure 2: Difference between traditional learning and transfer learning based approaches. (a) Traditional learning approach; (b) Transfer learning based approach.

NLP [13, 12, 42] based models for image and text classification respectively. The source code strictly follows the rules defined by their grammar 1 , thus these models are not suitable for our purpose. In this work, we first train two variants of recurrent neural networks-based models RNN and GRU for the purpose of transfer learning in the field of source code. We choose RNN and GRU because of their recent success in the modeling of source code. To train the models for transfer learning, we gather the data set used in previous studies [23, 38]. Table 1 shows the details of the data set used to build pre-trained models. By combining all collected projects, we end up with 13 million code tokens with a large vocabulary of size 177,342 .

Table 1: Data set used to pre-train models for transfer learning. The table shows name of the project, version of the project, line of code (LOC), total code tokens and unique code tokens found in each project.

\begin{tabular}{ccccc}
\hline Projects & Version & LOC & Total & Vocab Size $(V)$ \\
\hline ant & 1.10 .5 & 149,960 & 920,978 & 17,132 \\
cassandra & 3.11 .3 & 318,704 & 2734218 & 33,424 \\
db40 & 7.2 & 241,766 & $1,435,382$ & 20,286 \\
jgit & 5.1 .3 & 199,505 & $1,538,905$ & 20,970 \\
poi & 4.0 .0 & 387,203 & $2,876,253$ & 47,756 \\
maven & 3.6 .0 & 69,840 & 494,379 & 8,066 \\
batik & 1.10 .0 & 195,652 & $1,246,157$ & 21,964 \\
jts & 1.16 .0 & 91,387 & 611,392 & 11,903 \\
itext & 5.5 .13 & 161,185 & $1,164,362$ & 19,113 \\
antlr & 4.7 .1 & 56,085 & 407,248 & 6,813 \\
\hline Total & & $1,871,287$ & $13,429,274$ & 177,342 \\
\hline
\end{tabular}

${ }_{1}^{1}$ https://docs.oracle.com/javase/specs/jls/se7/html/jls-18.html 
Table 2: Deep learning models architecture summary used to pre-train source code models for transfer learning purpose.

\begin{tabular}{rrcc}
\hline & Type & Size & Activations \\
\hline Input & Code embedding & 300 & \\
Estimator & RNN,GRU & 300 & tanh \\
Over Fitting & Dropout & & \\
Output & Dense & $V$ & softmax \\
Loss & Categorical cross entropy & & \\
Optimizer & Adam & & \\
\hline
\end{tabular}

\subsubsection{Pre-Training Models for Transfer Learning}

All models are trained on Intel(R) Xeon(R) Silver $4110 \mathrm{CPU} 2.10 \mathrm{GHz} x$ 32 cores and 128GB of ram running Ubuntu 18.04.2 LTS operating system, equipped with the latest NVIDIA GeForce RTX 2080. The Table. 2 shows the architecture of trained models used for transfer learning. We follow the same approach used in previous works [56, 43] to pre-process the data set. To build a global vocabulary system, we remove all code tokens appearing less than three times in the collected data set which ends up with the vocabulary size of 88,022 unique code tokens. We map the vocabulary $(V)$ to a continuous feature vector of dense size 300. We use 300 hidden units with context size $(\tau)$ of 20 . For each model training we employ Adam optimizer with the default learn rate of 0.001. To control overfitting, we use Dropout. Each model is trained until it converges by employing early stop with the patience of three consecutive hits on the validation loss. One important thing to point out here is that the training process of these models is one time and do not need retraining. The trained models are publicly available ${ }^{2}$ for the purpose of transfer learning.

\subsection{Learning to Transfer Knowledge}

For transfer learning, we prepared the pre-trained models as described earlier in this section. Then, we use these pre-trained models to transfer the learned knowledge for the downstream task of source code suggestion. A key insight is to freeze the learned knowledge in pre-trained models to keep the learned knowledge unchanged and then fine-tune the extracted knowledge. In the proposed approach, we use the attention learner to fine-tune the model for the source code suggestion task. The attention learner pays attention to the task-specific features to achieve optimal performance. The Fig. 3 shows the architecture design of our proposed transfer learning-based attention model. We show the effectiveness of the proposed approach with the downstream task of source code suggestion. A source code suggestion engine recommends the next possible source code token given a context.

\footnotetext{
${ }^{2}$ Trained Models: https://github.com/yaxirhuxxain/TransferLearning
} 


\subsubsection{PreProcessing}

In this section, we briefly introduce each of the key preprocessing steps that we apply for the downstream task of source code suggestion. We perform normalization, tokenization and feature extraction. For the illustrating example, Table 3 shows the effect of each preprocessing step. We discuss each step in detail in the following subsections.

Table 3: An example of preprocessing steps.

\begin{tabular}{|c|c|}
\hline Original Source code & 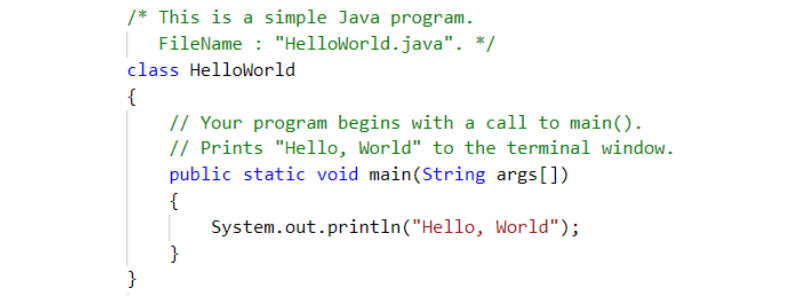 \\
\hline Normalized Source Code & 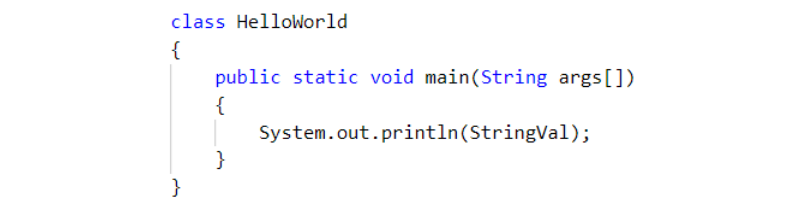 \\
\hline Tokenized Source Code & 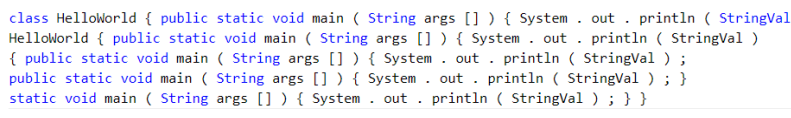 \\
\hline Vectorized Source Code & $\begin{array}{llllllllllllllllllll}1 & 2 & 3 & 4 & 5 & 6 & 7 & 8 & 9 & 10 & 11 & 12 & 3 & 13 & 14 & 15 & 14 & 16 & 8 & 17 \\
2 & 3 & 4 & 5 & 6 & 7 & 8 & 9 & 10 & 11 & 12 & 3 & 13 & 14 & 15 & 14 & 16 & 8 & 17 & 12 \\
3 & 4 & 5 & 6 & 7 & 8 & 9 & 10 & 11 & 12 & 3 & 13 & 14 & 15 & 14 & 16 & 8 & 17 & 12 & 18 \\
4 & 5 & 6 & 7 & 8 & 9 & 10 & 11 & 12 & 3 & 13 & 14 & 15 & 14 & 16 & 8 & 17 & 12 & 18 & 19 \\
5 & 6 & 7 & 8 & 9 & 10 & 11 & 12 & 3 & 13 & 14 & 15 & 14 & 16 & 8 & 17 & 12 & 18 & 19 & 19\end{array}$ \\
\hline
\end{tabular}

\section{Normalization}

One of the vital preprocessing steps is to normalize the data set. Usually, a data set contains some values which are unnecessary for a particular task, these type of values will intensely upset the outcome of the analysis. For this purpose, we normalize the source code files by removing all blank lines, inline and block-level comments. We replace all constant numerical values to their generic types (e.g. $1=$ IntVal, $1.2=$ FloatVal) and replace constant strings with a generic StringVal token.

\section{Tokenization}

After normalizing source code files, we tokenize the source code files. Tokenization is the process of extracting terms/words from the data set. For this 
purpose, each source code file is parsed into a sequence of space-separated code tokens. Each sequence is then parted into multiple subsequences of fixed size context 20 56.

\section{Vectorization}

To convert the source code sequences into a form that is suitable for training deep learning models we perform a series of transformations. First, we replace common tokens occurring only once in the corpus with a special token unk to build a global vocabulary system. Next, we build the vocabulary where each unique source code token corresponds to an entry in the vocabulary. Then each source code token is assigned a unique positive integer corresponding to its vocabulary index to convert the sequences (feature vectors) into a form that is suitable for training a deep learning model.

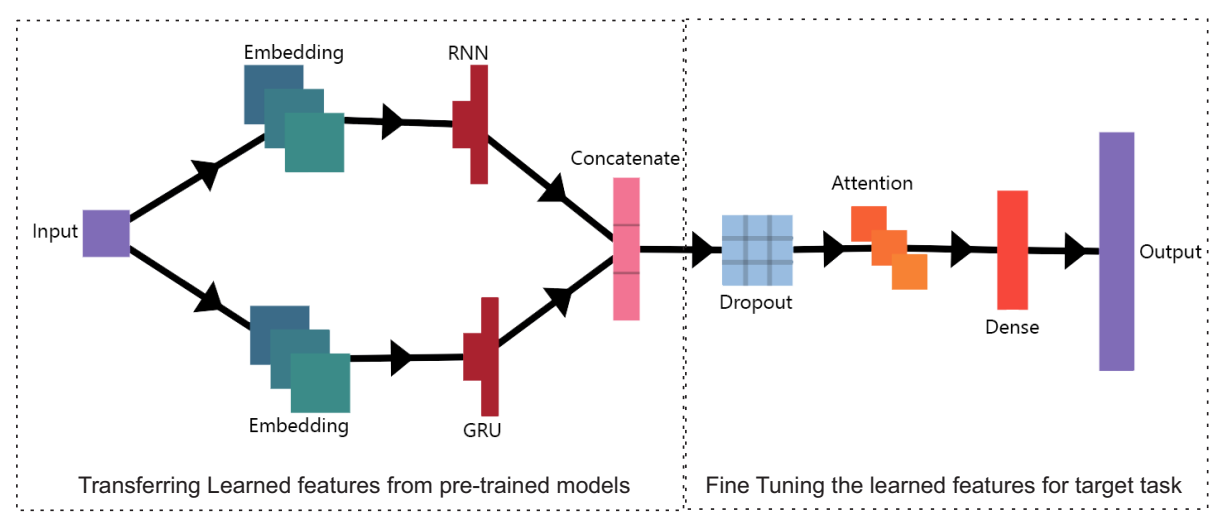

Figure 3: Proposed transfer learning based attention model architecture.

Table 4: Proposed transfer learning based attention model architecture summary.

\begin{tabular}{|c|c|c|c|c|}
\hline & Layers & Type & Size & Activations \\
\hline Frozen & $\begin{array}{l}\text { Input } \\
\text { Estimator } \\
\text { Combining }\end{array}$ & $\begin{array}{c}\text { Code embedding } \\
\text { RNN,GRU } \\
\text { Concatenate }\end{array}$ & $\begin{array}{l}300 \\
300\end{array}$ & tanh \\
\hline Fine Tuned & $\begin{array}{l}\text { Over Fitting } \\
\text { Attention } \\
\text { Output } \\
\text { Loss } \\
\text { Optimizer }\end{array}$ & $\begin{array}{c}\text { Dropout } \\
\text { Attention Learner } \\
\text { Dense } \\
\text { Categorical cross entropy } \\
\text { Adam }\end{array}$ & $V$ & softmax \\
\hline
\end{tabular}




\section{Evaluation}

In this section, we evaluate the effectiveness of our proposed approach by investigating the following research questions:

- RQ1: Does the proposed approach outperform the state-of-the-art approaches? if yes, to what extent?

- RQ2: How well the proposed approach performs in terms of source code suggestion task as compared to other baseline approaches?

- RQ3: Does normalization help to improve the performance of the proposed approach? If yes, to what extent?

To answer the research question (RQ1), we compare the performance of the proposed approach with the state-of-the-art approaches. To answer the research question (RQ2), we evaluate and compare the proposed approach for source code suggestion tasks with other baseline approaches. To answer the research question (RQ3), We conduct a comparative analysis to show the impact of normalization on model performance.

\subsubsection{Data set}

To empirically evaluate our work, we collected java projects from GitHub a well-known open-source software repositories provider. We gather the top five java projects sorted by the number of stars from GitHub at the time of this study. We download the latest snapshot of the project usually named as the master branch. Here, we choose the projects which are not used while training the pre-trained models discussed in Section 4. The Table 5 shows the version of each project, the total number of code lines, total code tokens and unique code tokens found in each project. To empirically evaluate our work, we repeat our experiment on each project separately. We randomly partition the projects into ten equal lines of code folds from which one fold is used for testing, one fold is used for model parameter optimization (validation) and rest of the folds are used for model training.

Table 5: List of projects used for the evaluation of this work.

\begin{tabular}{rrrrr}
\hline & & & \multicolumn{2}{c}{ Code Tokens } \\
Projects & Version & LOC & Total & Vocab Size $(V)$ \\
\hline elastic-search & $\mathrm{v} 7.0 .0$ & 210,357 & $1,765,479$ & 24,691 \\
java-design-patterns & $\mathrm{v} 1.20 .0$ & 30,784 & 200,344 & 5,649 \\
RxJava & $\mathrm{v} 2.2 .8$ & 257,704 & $1,908,258$ & 12,230 \\
interviews & $\mathrm{v} 1.0$ & 13,750 & 80,074 & 1,157 \\
spring-boot & $\mathrm{v} 2.2 .0 . \mathrm{M} 2$ & 224,465 & $1,813,891$ & 34,609 \\
\hline
\end{tabular}




\subsection{Training and Prediction}

We train several baseline models for the evaluation of this work. The proposed approach is evaluated in the following manner;

- We train RNN 43 based model as baseline similar to White et al. [56].

- We train GRU based deep neural model as baseline similar to Cho et al. [10]

- We train transfer learning-based attention model by following the proposed approach as discussed in Section 4.

We choose the approach proposed by White et al. [56] for comparison because they have shown the effectiveness of their approach with the similar task of source code suggestion and as far as we know, considered as the state-ofthe-art approach. We train the GRU [10] based model as the baseline because GRU based model is an advanced version of RNN which removes the vanishing gradient problem and performs better. The Table. 4 shows the proposed transfer learning-based attention model architecture. First, we preprocess the data set as discussed earlier in Section 4.2.1. Then, we map the vocabulary to a continuous feature vector of dense size 300 . We use 300 hidden units with context size $(\tau)$ of 20 . For each model training we employ Adam optimizer with the default learn rate of 0.001. To control overfitting, we use Dropout. Each

model is trained until it converges by employing early stop with the patience of three consecutive hits on the validation loss.

For the prediction of next source code token $y$ in a source code file, the model takes the context information prior to the prediction position $y$. Then, we use the trained models to predict the most likely next source code suggestions for the given context. If the predicted source code token is the same one as the original then we consider it a success.

\subsection{Metrics}

Usually deep learning approaches are evaluated by using different performance metrics. We choose similar evaluation approach as in previous studies. We choose top-k accuracy [56, 43] and Mean Reciprocal Rank (MRR) 38, 46] metrics for the evaluation of this work. Further, to evaluate the performance of the proposed approach we measure the precision, recall and F-measure scores which are widely used metrics [5]. Furthermore, to evaluate the significance of the proposed approach we perform ANOVA statistical testing. The computed metrics are formalized as,

$$
\begin{gathered}
\text { Accuracy }=\frac{T P+T N}{T P+F N+F P+T N} \\
\text { Precision }=\frac{T P}{T P+F P}
\end{gathered}
$$




$$
\begin{gathered}
\text { Recall }=\frac{T P}{T P+F N} \\
\text { F-measure }=2 * \frac{\text { Precision } * \text { Recall }}{\text { Precision }+ \text { Recall }}
\end{gathered}
$$

Where true positive (TP) defines the total number of source code suggestions that are predicted correctly by the model. The true negative (TN) defines the total number of source code suggestions that are predicted incorrectly by the model. The false positive (FP) defines the total number of source code suggestions that are mistakenly predicted correctly by the model. Similarly, the false negative (FN) defines the total number of source code suggestions that are mistakenly predicted incorrectly by the model.

\section{Results}

In this section, we will discuss and compare the results of our proposed approach with other baseline models.

\subsection{RQ1: Comparison against the baseline approaches}

The top-k accuracy score of the proposed approach and the baseline approaches are presented in Fig. 4. We make the following observations form Fig. 4

- The average accuracy rate of RNN based model is 45.01\%@ $k=1,65.56 \% @ k=5$ and 68.55\%@k=10, for the GRU based model is 50.06\%@k=1,64.38\%@k=5 and 73.27\%@k=10, while the proposed approach's average score is $66.15 \% @ k=1$, 90.68\%@k=5 and 93.97\%@k=10 which is much higher as compared to the baseline approaches.

- On average the proposed approach improves the accuracy ( $k @ 1$ ) by $21.14 \%$ from RNN and $16.09 \%$ from GRU based model.

- Results suggests that by employing the transfer learning-based attention model it significantly improves the model performance.

Further, to evaluate the performance of the proposed approach we measure the precision, recall and F-measure scores. Table 6 exhibits the precision, recall

and F-measure scores. From the Table 6 and Fig. 5, we make the following observations

- The proposed approach's average F-measure is 68.36 , while RNN and GRU gain much lower score of 39.73 and 46.20 respectively.

- The proposed approach's minimum F-measure is much higher than the maximum F-measure of the baseline approaches.

- The results suggest that the proposed approach outperforms the state-ofthe-art approaches in precision, recall, and F-measure. 
Table 6: Precision, Recall and F-measure comparison with baseline approaches

\begin{tabular}{|c|c|cc|c|}
\hline & & \multicolumn{2}{|c|}{ Baselines } & \\
& & RNN & GRU & Proposed \\
\hline \multirow{3}{*}{ elasticsearch } & Precision & 30.43 & 38.91 & $\mathbf{6 3 . 8 2}$ \\
& Recall & 40.75 & 46.86 & $\mathbf{8 2 . 8 9}$ \\
& F-measure & 34.84 & 42.52 & $\mathbf{7 2 . 1 1}$ \\
\hline \multirow{3}{*}{ spring-boot } & Precision & 37.29 & 40.77 & $\mathbf{6 2 . 3 9}$ \\
& Recall & 43.86 & 50.19 & $\mathbf{6 2 . 9 3}$ \\
& F-measure & 40.31 & 44.99 & $\mathbf{6 2 . 6 5}$ \\
\hline \multirow{3}{*}{ RxJava } & Precision & 41.15 & 46.54 & $\mathbf{6 6 . 9 7}$ \\
& Recall & 46.20 & 54.81 & $\mathbf{6 6 . 0 1}$ \\
& F-measure & 43.53 & 50.34 & $\mathbf{6 6 . 4 8}$ \\
\hline \multirow{3}{*}{ java-design } & Precision & 37.68 & 41.23 & $\mathbf{6 9 . 0 9}$ \\
& Recall & 41.76 & 48.08 & $\mathbf{6 8 . 7 1}$ \\
& F-measure & 39.62 & 44.39 & $\mathbf{6 8 . 8 9}$ \\
\hline \multirow{2}{*}{ interviews } & Precision & 38.11 & 47.00 & $\mathbf{7 1 . 0 2}$ \\
& Recall & 42.47 & 50.39 & $\mathbf{7 0 . 1 9}$ \\
& F-measure & 40.17 & 48.63 & $\mathbf{7 0 . 6 0}$ \\
\hline \multirow{2}{*}{ Average } & Precision & 36.93 & 42.89 & $\mathbf{6 6 . 6 6}$ \\
& Recall & 43.01 & 50.07 & $\mathbf{7 0 . 1 5}$ \\
& F-measure & 39.73 & 46.20 & $\mathbf{6 8 . 3 6}$ \\
\hline
\end{tabular}




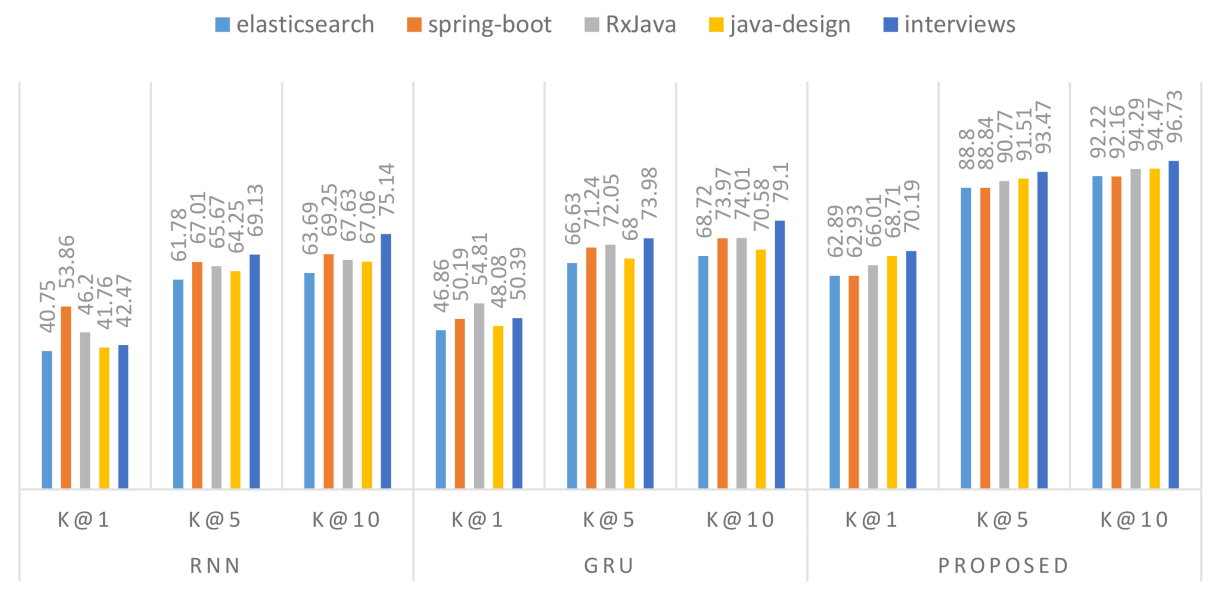

Figure 4: Top-k accuracy comparison.

\subsection{RQ2: Comparative analysis for Source Code Suggestion Task}

To further quantify the accuracy of the proposed approach for source code suggestion task, we measure the Mean Reciprocal Rank (MRR) scores of each model. The MRR is a rank-based evaluation metric which produces a value between $0-1$, where the value closer to 1 indicates a better source code suggestion model. The MRR can be expressed as

$$
\operatorname{MRR}(C)=\frac{1}{|C|} \sum_{i=1}^{|C|} \frac{1}{y^{i}}
$$

where $C$ is code sequence and $y^{i}$ refers to the index of the first relevant prediction. $M R R(C)$ is the average of all sequences $C$ in the test data set.

The results of all models are presented in the Table 7 . The average MRR score of RNN is 0.5156 and the average score of GRU is 0.5749 , while the average score of the proposed approach is 0.7618 which is much higher. From the results, we conclude that the proposed approach significantly outperforms the baseline approaches.

To further validate the statistical significance, we employ the ANOVA OneWay statistical test. We conduct the AVOVA test with its default settings ( $\alpha$ $=0.05$ ) using Microsoft Excel, and no modifications were made. Comparing (Table 8) the proposed approach with the best baseline (GRU), we found $F>$ $F$-crit and P-value $<\alpha$ is true in all cases (Accuracy, MRR, Precision, Recall and F-measure); therefore, we reject the null hypothesis, suggesting that using different approaches has statistically significant difference in performance.

\subsection{RQ3: Impact of Normalization}

The evaluation results of the proposed approach for normalized source code and non-normalized source code are presented in Table 9 . We only remove the 
Table 7: MRR scores with and without proposed approach

\begin{tabular}{|c|c|c|c|}
\hline & \multicolumn{2}{|c|}{ Baselines } & \multirow[b]{2}{*}{ Proposed } \\
\hline & $\mathrm{RNN}$ & $\overline{\mathrm{GR}} \mathrm{U}$ & \\
\hline elasticsearch & 0.4851 & 0.5405 & 0.7344 \\
\hline spring-boot & 0.5161 & 0.5672 & 0.7363 \\
\hline RxJava & 0.5403 & 0.6085 & 0.7619 \\
\hline java-design & 0.5082 & 0.5625 & 0.7805 \\
\hline interviews & 0.5284 & 0.5960 & 0.7960 \\
\hline Average & 0.5156 & 0.5749 & 0.7618 \\
\hline
\end{tabular}

Table 8: ANOVA Analysis.

\begin{tabular}{|c|c|c|c|c|c|c|}
\hline Source & $S S$ & $d f$ & $M S$ & $F$ & P-value & F-crit \\
\hline \multicolumn{7}{|l|}{ Accuracy (K@1) } \\
\hline Between Groups & 646.416 & 1 & 646.416 & 64.04975 & 4.35463E-05 & 5.317655 \\
\hline Within Groups & 80.73924 & 8 & 10.092405 & & & \\
\hline Total & 727.1552 & 9 & & & & \\
\hline \multicolumn{7}{|l|}{$M R R$} \\
\hline Between Groups & 646.416 & 1 & 646.416 & 64.04975 & 4.35463E-05 & 5.317655 \\
\hline Within Groups & 80.73924 & 8 & 10.092405 & & & \\
\hline Total & 727.1552 & 9 & & & & \\
\hline \multicolumn{7}{|l|}{ Precision } \\
\hline Between Groups & 1412.295 & 1 & 1412.29456 & 108.0003 & $6.36396 \mathrm{E}-06$ & 5.317655 \\
\hline Within Groups & 104.6141 & 8 & 13.07676 & & & \\
\hline Total & 1516.909 & 9 & & & & \\
\hline \multicolumn{7}{|l|}{ Recall } \\
\hline Between Groups & 1008.016 & 1 & 1008.016 & 29.81202 & 0.000601504 & 5.317655 \\
\hline Within Groups & 270.4992 & 8 & 33.812405 & & & \\
\hline Total & 1278.515 & 9 & & & & \\
\hline \multicolumn{7}{|l|}{ F-measure } \\
\hline Between Groups & 1206.92 & 1 & 1206.92196 & 99.9581 & $8.5015 \mathrm{E}-06$ & 5.31766 \\
\hline Within Groups & 96.5942 & 8 & 12.07428 & & & \\
\hline Total & 1303.52 & 9 & & & & \\
\hline
\end{tabular}




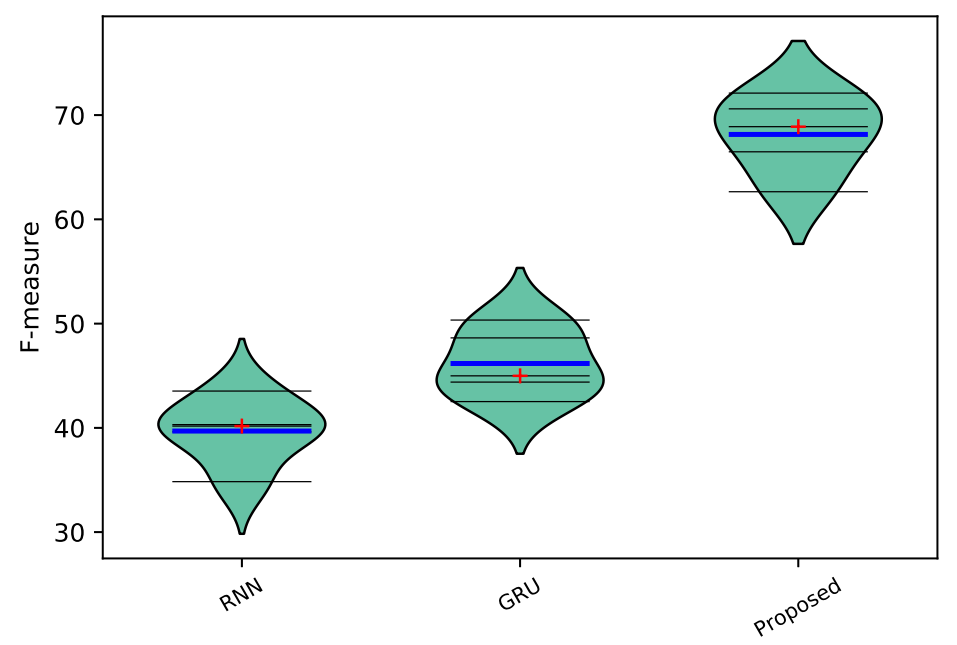

Figure 5: F-measure distribution.

comments while building the non-normalized source code. From the results, we observe that the normalization of source code improves the model performance significantly. On average the proposed approach with normalization achieves the accuracy score of $66.15 @ \mathrm{k}=1$ where without normalization the accuracy drops to $56.27 @ \mathrm{k}=1$. From the results (Table 9), we conclude that the normalization process significantly affects the model performance.

Table 9: Impact of Normalization

\begin{tabular}{|c|c|c|c|c|c|}
\hline & Accuracy & Precision & Recall & F-measure & MRR \\
\hline Normalized & 66.15 & 66.66 & 70.15 & 68.36 & 0.7618 \\
\hline Non-Normalized & 56.27 & 57.25 & 62.14 & 54.66 & 0.6524 \\
\hline
\end{tabular}

\subsection{Additional Findings}

From our experiments, we find several interesting facts. First, we notice that the transfer learning-based models take advantage of a large set of pretrained parameters resulting in a significant performance boost. Fig. 6 shows the parameters in each model and time per epoch. we can observe that the proposed approach's average parameters are 58M (million) which are much higher as compared to other baseline's average parameters which are $9 \mathrm{M}$. The parameters space of the proposed approach is much larger than the other baseline with minimum to none overhead on time. The proposed approach significantly boosts the model's performance by leveraging pre-trained knowledge without needing 
to learn the parameters from scratch. One important thing to mention here is that the model training is offline thus has no impact on the source code suggestion task. The proposed approach can suggest the next source code token in less than 20 milliseconds. Moreover, we experimented with another variant of the recurrent neural network named LSTM. We found that the performance of LSTM is worse as compared to RNN and GRU, thus we choose not to use it for transfer learning purpose. Furthermore, to evaluate the proposed approach qualitatively consider the example input code public static void display(int [] [] matrix) \{ System.out. , where the next possible source code token could be println. The proposed approach correctly captures the source code context and predicts the most probable next source code suggestions [println,print,writeShort], effectively ranking println on its first index.

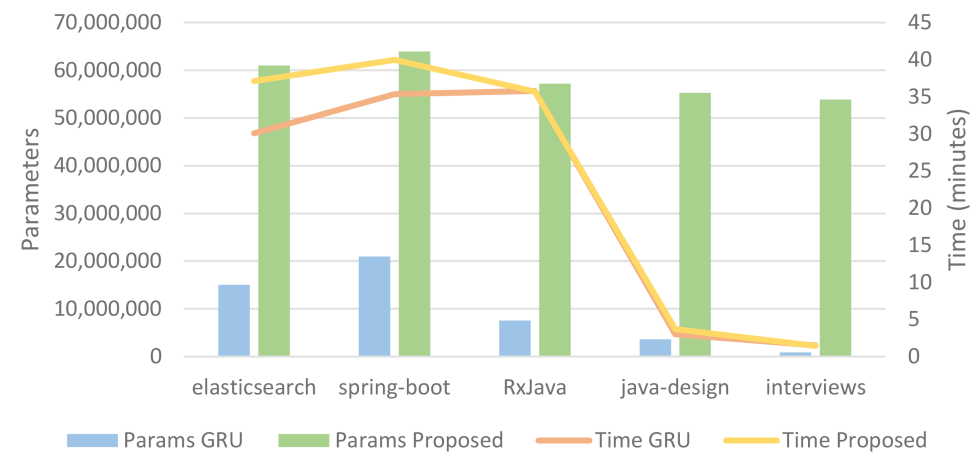

Figure 6: Model parameters and train time (epoch).

The proposed approach attains the finest performance due to several different reasons. First, the proposed approach takes leverage from pre-trained models by transferring the learned features from them. Second, the attention learner finetunes the model by paying attention to only task-specific features and does not increase the computational complexity which resulted in better performance. Consequently, transfer learning-based attention model has better generalization capability without training the model from scratch.

The broader impact of our work is to show that transfer learning could be beneficial in the domain of source code modeling. This work is the first step in this direction and results encourage future research on it. The work can be improved in several different ways. First, the performance of the proposed approach can be improved by hyper-parameter optimization [36. Second, the proposed approach can be improved by using complex architectures such as transformers [13] and stacked neural networks [53. Another possible path for improvement is to train the model on an even larger data set. In the future, we consider exploiting these possibilities. 


\section{Threats to Validity}

A risk to construct validity is the selection of assessment metrics. To alleviate this threat, we use several different evaluation metrics. We use the Top-k accuracy metric as done by former studies $223,56,38$. We use the precision, recall, and F-measure [5] metrics for the evaluation of the proposed approach. These metrics are most generally used for the model evaluation purpose. Moreover, we evaluate the proposed approach with MRR [38, 46] metric which is a ranked based metric. Further, to show the statistical significance of the proposed approach we adopt the ANOVA statistical testing.

A risk to internal validity is the employment of the baseline methods. We re-implement the baseline approaches by following the process described in the original manuscripts. To alleviate this risk, we twofold the implementations and results. Conversely, there could be some unobserved inaccuracies. Another risk is the choice of hyper-parameters for deep learning methods. The change in training, validation or testing set or the variation in hyper-parameters may impact the performance of the anticipated method.

A threat to external validity is related to the generality of results. The data set used in this study is collected from GitHub, a well-known source code repositories provider. It is not necessary that the projects used in this study represent other languages or Java language source code entirely.

\section{Conclusion}

In this work, we proposed a deep learning-based source code language model by using the concept of transfer learning. First, we exploit the concept of transfer learning for neural language-based source code models. Next, we presented RNN and GRU based pre-trained models for the purpose of transfer learning in the domain of source code. Both models are trained on over 13 million code tokens and do not need retraining and can directly be used for the purpose of transfer learning. We evaluated the proposed approach with the downstream task of source code suggestion. We evaluated the proposed approach extensively and compared it with the state-of-the-art models. The extensive evaluation of this work suggests that the proposed approach significantly improves the model's performance by exploiting the concept of transfer learning.

\section{Acknowledgments}

This work was supported by the National Key R\&D (grant no. 2018YFB1003902), Natural Science Foundation of Jiangsu Province (No. BK20170809), National Natural Science Foundation of China (No. 61972197) and Qing Lan Project.

\section{References}

[1] Miltiadis Allamanis and Charles Sutton. Mining idioms from source code. In Proceedings of the 22nd ACM SIGSOFT International Symposium on Foundations of Software Engineering, pages 472-483. ACM, 2014. 
[2] Miltiadis Allamanis, Earl T Barr, Christian Bird, and Charles Sutton. Suggesting accurate method and class names. In Proceedings of the 2015 10th Joint Meeting on Foundations of Software Engineering, pages 38-49. ACM, 2015.

[3] Miltiadis Allamanis, Hao Peng, and Charles Sutton. A convolutional attention network for extreme summarization of source code. In International Conference on Machine Learning, pages 2091-2100, 2016.

[4] Miltos Allamanis, Daniel Tarlow, Andrew Gordon, and Yi Wei. Bimodal modelling of source code and natural language. In International conference on machine learning, pages 2123-2132, 2015.

[5] Uri Alon, Meital Zilberstein, Omer Levy, and Eran Yahav. code2vec: Learning distributed representations of code. Proceedings of the ACM on Programming Languages, 3(POPL):40, 2019.

[6] Dzmitry Bahdanau, Jan Chorowski, Dmitriy Serdyuk, Philemon Brakel, and Yoshua Bengio. End-to-end attention-based large vocabulary speech recognition. In 2016 IEEE international conference on acoustics, speech and signal processing (ICASSP), pages 4945-4949. IEEE, 2016.

[7] Pavol Bielik, Veselin Raychev, and Martin Vechev. Phog: probabilistic model for code. In International Conference on Machine Learning, pages 2933-2942, 2016.

[8] Wing-Kwan Chan, Hong Cheng, and David Lo. Searching connected api subgraph via text phrases. In Proceedings of the ACM SIGSOFT 20th International Symposium on the Foundations of Software Engineering, page 10. ACM, 2012.

[9] Chunyang Chen and Zhenchang Xing. Similartech: automatically recommend analogical libraries across different programming languages. In 2016 31st IEEE/ACM International Conference on Automated Software Engineering (ASE), pages 834-839. IEEE, 2016.

[10] Kyunghyun Cho, Bart Van Merriënboer, Caglar Gulcehre, Dzmitry Bahdanau, Fethi Bougares, Holger Schwenk, and Yoshua Bengio. Learning phrase representations using rnn encoder-decoder for statistical machine translation. arXiv preprint arXiv:1406.1078, 2014.

[11] Jan K Chorowski, Dzmitry Bahdanau, Dmitriy Serdyuk, Kyunghyun Cho, and Yoshua Bengio. Attention-based models for speech recognition. In Advances in neural information processing systems, pages 577-585, 2015.

[12] Zihang Dai, Zhilin Yang, Yiming Yang, William W Cohen, Jaime Carbonell, Quoc V Le, and Ruslan Salakhutdinov. Transformer-xl: Attentive language models beyond a fixed-length context. arXiv preprint arXiv:1901.02860, 2019. 
[13] Jacob Devlin, Ming-Wei Chang, Kenton Lee, and Kristina Toutanova. Bert: Pre-training of deep bidirectional transformers for language understanding. arXiv preprint arXiv:1810.04805, 2018.

[14] Andrea Renika DSouza, Di Yang, and Cristina V Lopes. Collective intelligence for smarter api recommendations in python. In 2016 IEEE 16th International Working Conference on Source Code Analysis and Manipulation (SCAM), pages 51-60. IEEE, 2016.

[15] Lixin Duan, Ivor W Tsang, Dong Xu, and Stephen J Maybank. Domain transfer svm for video concept detection. pages 1375-1381, 2009.

[16] Jaroslav Fowkes, Pankajan Chanthirasegaran, Razvan Ranca, Miltiadis Allamanis, Mirella Lapata, and Charles Sutton. Autofolding for source code summarization. IEEE Transactions on Software Engineering, 43(12):1095$1109,2017$.

[17] Christine Franks, Zhaopeng Tu, Premkumar Devanbu, and Vincent Hellendoorn. Cacheca: A cache language model based code suggestion tool. In Proceedings of the 37th International Conference on Software EngineeringVolume 2, pages 705-708. IEEE Press, 2015.

[18] Xiaodong Gu, Hongyu Zhang, Dongmei Zhang, and Sunghun Kim. Deep api learning. In Proceedings of the 2016 24th ACM SIGSOFT International Symposium on Foundations of Software Engineering, pages 631-642. ACM, 2016.

[19] Latifa Guerrouj, David Bourque, and Peter C Rigby. Leveraging informal documentation to summarize classes and methods in context. In 2015 IEEE/ACM 37th IEEE International Conference on Software Engineering, volume 2, pages 639-642. IEEE, 2015.

[20] Rahul Gupta, Aditya Kanade, and Shirish Shevade. Deep reinforcement learning for programming language correction. arXiv preprint arXiv:1801.10467, 2018.

[21] Kaiming He, Xiangyu Zhang, Shaoqing Ren, and Jian Sun. Deep residual learning for image recognition. In Proceedings of the IEEE conference on computer vision and pattern recognition, pages 770-778, 2016.

[22] Vincent J Hellendoorn and Premkumar Devanbu. Are deep neural networks the best choice for modeling source code? In Proceedings of the 2017 11th Joint Meeting on Foundations of Software Engineering, pages 763773. ACM, 2017.

[23] Abram Hindle, Earl T Barr, Zhendong Su, Mark Gabel, and Premkumar Devanbu. On the naturalness of software. In 2012 34th International Conference on Software Engineering (ICSE), pages 837-847. IEEE, 2012. 
[24] Junlin Hu, Jiwen Lu, and Yap-Peng Tan. Deep transfer metric learning. In Proceedings of the IEEE conference on computer vision and pattern recognition, pages 325-333, 2015.

[25] Zhongling Huang, Zongxu Pan, and Bin Lei. Transfer learning with deep convolutional neural network for sar target classification with limited labeled data. Remote Sensing, 9(9):907, 2017.

[26] Yasir Hussain, Zhiqiu Huang, Senzhang Wang, and Yu Zhou. Codegru: Context-aware deep learning with gated recurrent unit for source code modeling. arXiv preprint arXiv:1903.00884, 2019.

[27] Yasir Hussain, Zhiqiu Huang, Yu Zhou, and Senzhang Wang. Deepvs: An efficient and generic approach for source code modeling usage. arXiv preprint arXiv:1910.06500, 2019.

[28] Srinivasan Iyer, Ioannis Konstas, Alvin Cheung, and Luke Zettlemoyer. Summarizing source code using a neural attention model. In Proceedings of the 54th Annual Meeting of the Association for Computational Linguistics (Volume 1: Long Papers), pages 2073-2083, 2016.

[29] He Jiang, Najam Nazar, Jingxuan Zhang, Tao Zhang, and Zhilei Ren. Prst: A pagerank-based summarization technique for summarizing bug reports with duplicates. International Journal of Software Engineering and Knowledge Engineering, 27(06):869-896, 2017.

[30] Iman Keivanloo, Juergen Rilling, and Ying Zou. Spotting working code examples. In Proceedings of the 36th International Conference on Software Engineering, pages 664-675. ACM, 2014.

[31] SanaUllah Khan, Naveed Islam, Zahoor Jan, Ikram Ud Din, and Joel JP C Rodrigues. A novel deep learning based framework for the detection and classification of breast cancer using transfer learning. Pattern Recognition Letters, 125:1-6, 2019.

[32] Mathias Kraus and Stefan Feuerriegel. Decision support from financial disclosures with deep neural networks and transfer learning. Decision Support Systems, 104:38-48, 2017.

[33] Balwinder Kumar and Satwinder Singh. Code clone detection and analysis using software metrics and neural network-a literature review. Complexity, $1(2): 3,2015$.

[34] Minh-Thang Luong, Hieu Pham, and Christopher D Manning. Effective approaches to attention-based neural machine translation. arXiv preprint arXiv:1508.04025, 2015.

[35] Chris Maddison and Daniel Tarlow. Structured generative models of natural source code. In International Conference on Machine Learning, pages 649-657, 2014. 
[36] Pawel Matuszyk, Renê Tatua Castillo, Daniel Kottke, and Myra Spiliopoulou. A comparative study on hyperparameter optimization for recommender systems. In Workshop on Recommender Systems and Big Data Analytics (RS-BDA'16).2016.S, pages 13-21, 2016.

[37] Anh Tuan Nguyen, Tung Thanh Nguyen, Hoan Anh Nguyen, Ahmed Tamrawi, Hung Viet Nguyen, Jafar Al-Kofahi, and Tien N Nguyen. Graphbased pattern-oriented, context-sensitive source code completion. In 2012 34th International Conference on Software Engineering (ICSE), pages 6979. IEEE, 2012.

[38] Anh Tuan Nguyen, Trong Duc Nguyen, Hung Dang Phan, and Tien N Nguyen. A deep neural network language model with contexts for source code. In 2018 IEEE 25th International Conference on Software Analysis, Evolution and Reengineering (SANER), pages 323-334. IEEE, 2018.

[39] Sinno Jialin Pan, James T Kwok, Qiang Yang, et al. Transfer learning via dimensionality reduction. In $A A A I$, volume 8, pages 677-682, 2008.

[40] Hung Viet Pham, Phong Minh Vu, Tung Thanh Nguyen, et al. Learning api usages from bytecode: a statistical approach. In Proceedings of the 38th International Conference on Software Engineering, pages 416-427. ACM, 2016.

[41] Maxim Rabinovich, Mitchell Stern, and Dan Klein. Abstract syntax networks for code generation and semantic parsing. arXiv preprint arXiv:1704.07535, 2017.

[42] Alec Radford, Jeffrey Wu, Rewon Child, David Luan, Dario Amodei, and Ilya Sutskever. Language models are unsupervised multitask learners. OpenAI Blog, 1(8), 2019.

[43] Veselin Raychev, Martin Vechev, and Eran Yahav. Code completion with statistical language models. In Acm Sigplan Notices, volume 49, pages 419-428. ACM, 2014.

[44] Veselin Raychev, Pavol Bielik, Martin Vechev, and Andreas Krause. Learning programs from noisy data. In ACM SIGPLAN Notices, volume 51, pages 761-774. ACM, 2016.

[45] Milad Salem, Shayan Taheri, and Jiann-Shiun Yuan. Utilizing transfer learning and homomorphic encryption in a privacy preserving and secure biometric recognition system. Computers, 8(1):3, 2019.

[46] Eddie Antonio Santos, Joshua Charles Campbell, Dhvani Patel, Abram Hindle, and José Nelson Amaral. Syntax and sensibility: Using language models to detect and correct syntax errors. In 2018 IEEE 25th International Conference on Software Analysis, Evolution and Reengineering (SANER), pages 311-322. IEEE, 2018. 
[47] Akshay Sethi, Anush Sankaran, Naveen Panwar, Shreya Khare, and Senthil Mani. Dlpaper2code: Auto-generation of code from deep learning research papers. In Thirty-Second AAAI Conference on Artificial Intelligence, 2018.

[48] Hoo-Chang Shin, Holger R Roth, Mingchen Gao, Le Lu, Ziyue Xu, Isabella Nogues, Jianhua Yao, Daniel Mollura, and Ronald M Summers. Deep convolutional neural networks for computer-aided detection: Cnn architectures, dataset characteristics and transfer learning. IEEE transactions on medical imaging, 35(5):1285-1298, 2016.

[49] Christian Szegedy, Wei Liu, Yangqing Jia, Pierre Sermanet, Scott Reed, Dragomir Anguelov, Dumitru Erhan, Vincent Vanhoucke, and Andrew Rabinovich. Going deeper with convolutions. In Proceedings of the IEEE conference on computer vision and pattern recognition, pages 1-9, 2015.

[50] Ferdian Thung, Shaowei Wang, David Lo, and Julia Lawall. Automatic recommendation of api methods from feature requests. In Proceedings of the 28th IEEE/ACM International Conference on Automated Software Engineering, pages 290-300. IEEE Press, 2013.

[51] Zhaopeng Tu, Zhendong Su, and Premkumar Devanbu. On the localness of software. In Proceedings of the 22nd ACM SIGSOFT International Symposium on Foundations of Software Engineering, pages 269-280. ACM, 2014.

[52] Ashish Vaswani, Noam Shazeer, Niki Parmar, Jakob Uszkoreit, Llion Jones, Aidan N Gomez, Łukasz Kaiser, and Illia Polosukhin. Attention is all you need. In Advances in neural information processing systems, pages 5998$6008,2017$.

[53] Pascal Vincent, Hugo Larochelle, Isabelle Lajoie, Yoshua Bengio, and Pierre-Antoine Manzagol. Stacked denoising autoencoders: Learning useful representations in a deep network with a local denoising criterion. Journal of machine learning research, 11(Dec):3371-3408, 2010.

[54] Jue Wang, Yingnong Dang, Hongyu Zhang, Kai Chen, Tao Xie, and Dongmei Zhang. Mining succinct and high-coverage api usage patterns from source code. In Proceedings of the 10th Working Conference on Mining Software Repositories, pages 319-328. IEEE Press, 2013.

[55] Senzhang Wang, Xia Hu, Philip S Yu, and Zhoujun Li. Mmrate: inferring multi-aspect diffusion networks with multi-pattern cascades. In Proceedings of the 20th ACM SIGKDD international conference on Knowledge discovery and data mining, pages 1246-1255. ACM, 2014.

[56] Martin White, Christopher Vendome, Mario Linares-Vásquez, and Denys Poshyvanyk. Toward deep learning software repositories. In Proceedings of the 12th Working Conference on Mining Software Repositories, pages 334-345. IEEE Press, 2015. 
[57] Tao Xie and Jian Pei. Mapo: Mining api usages from open source repositories. In Proceedings of the 2006 international workshop on Mining software repositories, pages 54-57. ACM, 2006.

[58] Tom Young, Devamanyu Hazarika, Soujanya Poria, and Erik Cambria. Recent trends in deep learning based natural language processing. ieee Computational intelligenCe magazine, 13(3):55-75, 2018.

[59] Yuan Yuan, Xiangtao Zheng, and Xiaoqiang Lu. Hyperspectral image superresolution by transfer learning. IEEE Journal of Selected Topics in Applied Earth Observations and Remote Sensing, 10(5):1963-1974, 2017.

[60] Wenlu Zhang, Rongjian Li, Tao Zeng, Qian Sun, Sudhir Kumar, Jieping Ye, and Shuiwang Ji. Deep model based transfer and multi-task learning for biological image analysis. IEEE transactions on Big Data, 2016.

[61] Xiaoming Zhang, Xiaoming Chen, Yan Chen, Senzhang Wang, Zhoujun Li, and Jiali Xia. Event detection and popularity prediction in microblogging. Neurocomputing, 149:1469-1480, 2015.

[62] Yu Zhou, Xin Yan, Wenhua Yang, Taolue Chen, and Zhiqiu Huang. Augmenting java method comments generation with context information based on neural networks. Journal of Systems and Software, 156:328-340, 2019. 